\title{
ON SYSTOLES AND ORTHO SPECTRUM RIGIDITY
}

\author{
HIDETOSHI MASAI AND GREG MCSHANE
}

\begin{abstract}
We consider the ortho spectrum of hyperbolic surfaces with totally geodesic boundary. We show that in general the ortho spectrum does not determine the systolic length but that there are only finitely many possibilities. As a corollary we show that, up to isometry, there are only finitely many hyperbolic structures on a surface that share a given ortho spectrum.
\end{abstract}

\section{INTRODUCTION}

1.1. Context. Mark Kac famously asked if one could hear the shape of a drum (c.f. [Gor89]). This has a more precise formulation namely: does the spectrum of the Laplacian of a closed hyperbolic surface determine the metric up to isometry? Huber and Selberg (c.f. [Bus10]) had shown that for such surfaces the Laplacian's spectrum is determined by the length spectrum which is the set of lengths of the closed geodesics counted with multiplicities. Examples were found of isospectral pairs that is hyperbolic structures $X, Y$ on a closed surface $\Sigma$ which have the same length spectrum but are not isometric and so Kac's question was answered in the negative. In particular, there is a very natural construction using finite covers due to Sunada ([Sun85], see also [Bus10] which allows one to construct many such pairs of surfaces.

Kac's original question about analytic data associated to a metric (the Laplacian) was translated to a question about metric data (the length of geodesics) and solved, at least partially, using what is essentially algebra. We say "partially" because it seems reasonable to conjecture that any pair of isospectral surfaces are commensurable that is they share a common finite cover.

Motivated by Kac's question, we consider an analogous problem. Basmajian [Bas93] defined the ortho spectrum of a hyperbolic structure $X$ on a surface $\Sigma$ of finite type with non empty (totally geodesic) boundary. He proved a formula for the perimeter, which we define to be the sum of the lengths of the boundary components, in terms of the ortho spectrum. Subsequently, Bridgeman and Kahn Bri11, BK10] gave a formula for the area of the surface in terms of the ortho spectrum. 
Thus two of the principal moduli of the hyperbolic structure $X$ are explicitly determined by the ortho spectrum.

It turns out that when one replaces length spectrum by ortho spectrum, the resulting analogue of Kac's question is easier to answer in the negative. This is because it is far easier to construct the required isospectral pairs for the ortho spectrum by using finite covers than for the length spectrum. So we set out to understand to what extent the ortho spectrum determines the geometry of the hyperbolic structure $X$ knowing that three important numerical invariants (moduli) of $X$ are determined by the ortho spectrum:

- The total length of the boundary by Basmajian [Bas93.

- The area of the surface by Bridgeman Bri11.

- The entropy of the geodesic flow can be determined via Poincaré series by J. Parker Par95].

Our point of view is much influenced by the work of Buser and his students who have studied Kac's original question in an attempt to give a complete answer. In fact Buser and Semmler [BS88] proved that for a one holed torus the spectrum does determine a hyperbolic structure up to isometry. Of fundamental importance in Buser's work is the systolic length which does not appear on our list of moduli explicitly determined by the ortho spectrum. Recall that the systolic length is the length of the shortest closed geodesic and it is easy to see that this is the infimum of the length spectrum. The systolic length is obviously a measure of how thin a surface gets but the Collar Lemma tells us that a short geodesic lives in wide collar so it also gives a lower bound on the diameter of a surface.

1.2. Statement of Results. Though the relationship between systolic length and length spectrum is quite straightforward its relationship to the ortho spectrum is less obvious and this is the main subject of this paper. We show using abelian covers (Theorem 6.3) that in general the ortho spectrum does not determine the systolic length but that (Theorem 5.1) there are only finitely many possibilities for the the systolic length for a given ortho spectrum. As a corollary we show that, up to isometry, there are only finitely many hyperbolic structures on a surface $\Sigma$ that share a given ortho spectrum. To do this, following Wolpert [Wol77], we use Mumford's pre compactness criterion for subsets of moduli space $\mathcal{M}(\Sigma)$ : a subset $U \subset \mathcal{M}(\Sigma)$ is pre compact, if and only if, the infimum of the systole over $U$ is strictly positive. Note here that when the surface $\Sigma$ has boundary, hyperbolic structures on $\Sigma$ we consider are assumed that all the components of the boundary are totally geodesic. Moreover we define the moduli space $\mathcal{M}(\Sigma)$ of $\Sigma$ 
to be the space of hyperbolic structures on $\Sigma$ with the fixed perimeter. Thus we must show that given a hyperbolic structure $X$ then there is a uniform lower bound on the systolic length which only depends on the ortho spectrum of $X$ and its topological complexity. Here, by topological complexity we mean the number of pairs of pants in a pants decomposition (equivalently the area of $X$ ). At the outset we hoped to obtain this result by using properties of the Poincaré series. Unfortunately, our argument (see Paragraph 5.3 for a detailed discussion) only worked when the entropy of the geodesic flow was strictly less than $\frac{1}{2}$. This is problematic since many surfaces do not satisfy this hypothesis and indeed when a surface has cusps the entropy is at least $\frac{1}{2}$. We did however obtain some partial results which we include here as they may be of independent interest (see Paragraphs 5.2 and 5.3). Notably we show using the identities and a geometric limit argument that for a finite area $n$-punctured hyperbolic surface with totally geodesic boundary which is topologically a punctured disc, there are only finitely many isospectral pairs. Finally, in Paragraph 5.4 we give a proof using a more combinatorial approach and some hyperbolic trigonometry which can in principle be made effective i.e. used to give an upper bound on the number of isospectral pairs for a given surface.

1.3. Questions, remarks, further work. For a compact surface without boundary Buser [Bus10, Chapter 13] has in fact given upper bounds in terms of the genus $g$ for the number of hyperbolic structures on a surface $\Sigma$ that share a given length spectrum. These bounds were exponential of the square of $g$, namely $e^{720 g^{2}}$. They have recently been much improved by Parlier Par18 to $g^{154 g}$. Since our proof depends on a compactness argument we do not obtain any such bounds. It seems an interesting question to render our theorem uniform that is to find a bound on the number of hyperbolic structures on a surface $\Sigma$ that share a given ortho spectrum purely in terms of the topological complexity of $\Sigma$ as defined above.

In Section 6 we give examples of hyperbolic structures on a surface $\Sigma$ with the same ortho spectrum but different systolic length. Since the systolic length is the infimum of the length spectrum they have different length spectra. At the time of writing, all known pairs of hyperbolic structures with the same length spectrum are commensurable (it is conjectured that this is always the case) so that, by covering theory (see Section 6) they necessarily have the same ortho spectrum. This suggests that the ortho spectrum is potentially a strictly weaker invariant than the length spectrum: i.e. the length spectrum determines the ortho spectrum but not vice versa. 
Finally, Bridgeman [Bri11] has investigated the ortho spectrum for an ideal polygon in the hyperbolic plane, showing how to obtain certain celebrated identities for the Rogers' dilogarithm from it. Together with Dumas [BD07] he went on to study a related problem namely that of the chord length distribution for the ideal triangle. In a forthcoming paper [MM] we consider the problem of whether the ortho spectrum determines an ideal polygon up to congruence.

Acknowledgement. Main part of this work was done during the visit of the second named author to Tokyo in early summer of 2019. The visit was supported by JSPS KAKENHI Grant Number $16 \mathrm{H} 02145$ and 19K14525. We would like to thank Ara Basmajian, Martin Bridgeman, Yi Huang, and Hugo Parlier for helpful conversations. We thank in particular Dragomir Saric whose comments helped improve exposition of the geometric limit arguments in 5.2. Finally, we would also like to thank the anonymous referees for helpful comments that improved the paper greatly.

\section{ORtho GEODESICS}

It is convenient to define ortho geodesics by using the action of a Fuchsian group on the universal cover of $\Sigma$. A Fuchsian group $\Gamma$ is a discrete subgroup of isom ${ }^{+}(\mathbb{H})$. If $\Gamma$ is torsion free then the quotient of $\mathbb{H}$ by the action of $\Gamma$ is a surface $\Sigma=\mathbb{H} / \Gamma$ and $\pi_{1}(\Sigma) \simeq \Gamma$. The limit set $\Lambda(\Gamma)$ of $\Gamma$ is the smallest closed $\Gamma$-invariant subset of the ideal boundary $\partial \mathbb{H}$ and, provided $\Gamma$ is not virtually abelian, this is a perfect set. The complement $\Omega(\Gamma)$ of the limit set is called the regular set if it is a (possibly empty) $\Gamma$-invariant open set. Further, if $\Gamma$ is finitely generated and $\Sigma$ does not have finite area then $\Omega(\Gamma)$ is dense and consists of countably many open intervals. If $\Gamma$ contains no parabolic elements then the orbits of the action of $\Gamma$ on $\Omega(\Gamma)$ are in 1-1 correspondence with the ends of $\Sigma$. Thus, we have a $\Gamma$-invariant decomposition of the ideal boundary of $\mathbb{H}$ as

$$
\partial \mathbb{H}=\Lambda(\Gamma) \sqcup \Omega(\Gamma) .
$$

We shall denote by $\partial \Omega$ the set of all the points $a, b$ such that the intersection of the interval $[a, b] \subset \partial \mathbb{H}$ with the limit set $\Lambda$ is $\{a, b\}$.

2.1. Convex core and ortho geodesics. Given $\Gamma$ finitely generated and $\Sigma$ of infinite area there is a canonical way to associate a subsurface $C(\Sigma) \subset \Sigma$ of finite area with totally geodesic boundary called the convex core. Let $C(\Lambda) \subset \mathbb{H}$ be the convex hull of the limit set, this is a closed, $\Gamma$-invariant subset whose frontier consists of countably many complete geodesics. The quotient $C(\Sigma):=C(\Lambda) / \Gamma$ embeds naturally 
into $\Sigma=\mathbb{H} / \Gamma$. By construction, $C(\Lambda)$ is the universal cover of $\Sigma$ and the embedding induces an isomorphism between $\pi_{1}(\Sigma) \simeq \Gamma$ and $\pi_{1}(C(\Sigma))$. In particular:

Proposition 2.1. The components of the regular set, i.e. the maximal intervals in the complement of $\Lambda$, are in 1-1 correspondence with lifts of the boundary geodesics of $\Sigma$.

Note that if $\gamma \subset \mathbb{H}$ is a geodesic with an endpoint in $\partial \Omega$ then the corresponding geodesic in the surface $\Sigma$ contains some boundary geodesic in its closure. In what follows we will think of $C(\Lambda)$ as a generalized polygon and refer to the geodesics of $\partial C(\Lambda)$ as sides. We associate to pairs of distinct sides an ortho geodesic $\hat{\alpha}^{*}$, which is just the unique common perpendicular joining the sides. The image of $\hat{\alpha}^{*}$ is a geodesic arc $\alpha^{*}$ which meets $\partial C(\Sigma)$ perpendicularly and this is an ortho geodesic on the surface $\Sigma$. By definition, the lengths of $\hat{\alpha}^{*}$ and $\alpha^{*}$ are the same and clearly the length of $\hat{\alpha}^{*}$ can be computed as a cross ratio of the endpoints of the associated sides of $C(\Lambda)$. We denote by $\mathcal{O}(\Sigma)$ the ortho spectrum of $\Sigma$, namely, the set of lengths of ortho geodesics counted with multiplicity.

2.2. Identities for moduli. One can compute two important numerical invariants (moduli) of the hyperbolic structure from the ortho spectrum, namely the perimeter (total length of the boundary) and the area.

Theorem 2.2 (Basmajian Bas93], Calegari Cal10]). For each $n \in \mathbb{N}$, there exists a function $B_{n}: \mathbb{R}_{>0} \rightarrow \mathbb{R}_{>0}$ with the following property. Let $M$ be a compact hyperbolic n-manifold with totally geodesic boundary. Then

$$
\operatorname{Vol}(\partial M)=\sum_{\ell \in \mathcal{O}(M)} B_{n}(\ell)
$$

If $\Sigma$ is a surface with a single totally geodesic boundary component $\delta$. Then the above formula can be written explicitly by

$$
\sum_{\alpha^{*}} 2 \sinh ^{-1}\left(\frac{1}{\sinh \left(\ell\left(\alpha^{*}\right)\right)}\right)=\ell(\delta) .
$$

Theorem 2.3 (Bridgeman-Kahn [BK10], Calegari [Cal10]). For each $n \in \mathbb{N}$, there exists a function $F_{n}: \mathbb{R}_{>0} \rightarrow \mathbb{R}_{>0}$ with the following property. Let $M$ be a compact hyperbolic $n$-manifold with totally geodesic boundary. Then

$$
\operatorname{Vol}(M)=\sum_{\ell \in \mathcal{O}(M)} F_{n}(\ell)
$$


Remark 2.4. The proofs in [BK10] and [Cal10] only use the fact that generic geodesics eventually meet the boundary. Hence their proof work not only for compact case but also the case where $M$ has cusps. We use the cusped version later in Section 5.2.

Remark 2.5. Bridgeman-Kahn and Calegari gave different proofs of Theorem 2.3. Given that the construction of Bridgeman-Kahn and of Calegari are different, so could be the functions $F_{n}$, but in [MM13 we show that these functions are the same.

\section{Geometry of PAirs of PANts}

This section contains material necessary in the proof of Theorem 5.1 below in particular certain relations between lengths of ortho geodesics in a pair of pants and the boundary lengths.

We begin by recalling a useful formula from hyperbolic trigonometry (see for example Bea95, Equation 7.18.2]) . Choose a pair of adjacent sides in a right angled pentagon and let $a, b$ denote their respective lengths (see Figure 1) then

$$
\cosh d=\sinh a \sinh b .
$$

where $d$ is the length of the unique side not adjacent to either of our chosen pair of sides.

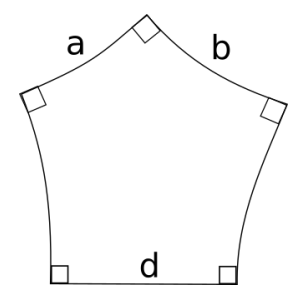

FIGURE 1

Definition 3.1. A foot of an ortho geodesic is a point of intersection of the ortho geodesic with the (totally geodesic) boundary of the surface. Since the surface is convex an ortho geodesic has exactly two feet.

Lemma 3.2. Let $P$ be a pair of pants with boundary geodesics $\alpha, \beta, \gamma$. Let $\tau$ be the unique simple ortho geodesic with both their feet on $\gamma$. Then after possibly exchanging the labels on $\alpha, \beta$, we have

$$
\sinh (\ell(\tau) / 2) \leq \frac{\cosh (\ell(\alpha) / 2)}{\sinh (\ell(\gamma) / 4)}
$$


Proof. The feet of $\tau$ divide $\gamma$ into two intervals one of which is of length $y \geq \ell(\gamma) / 2$. After possibly exchanging the labels on $\alpha, \beta$ one may assume that there is an embedded right angled pentagon in $P$ with a pair of adjacent sides of length $y / 2, \ell(\tau) / 2$ and the non adjacent side has length $\ell(\alpha) / 2$. Applying formula (1) one obtains

$$
\cosh (\ell(\alpha) / 2)=\sinh (y / 2) \sinh (\ell(\tau) / 2) \geq \sinh (\ell(\gamma) / 4) \sinh (\ell(\tau) / 2) .
$$

So

$$
\sinh (\ell(\tau) / 2) \leq \frac{\cosh (\ell(\alpha) / 2)}{\sinh (\ell(\gamma) / 4)}
$$

Lemma 3.3. Let $P$ be a pair of pants with boundary geodesics $\alpha, \beta, \gamma$. Let $\tau$ and $\tau^{\prime}$ be ortho geodesics with both their feet on $\gamma$ such that $\tau$ is simple and $\tau^{\prime}$ goes round $\alpha$ exactly once (see Figure 2). Then we have

$$
\begin{aligned}
\cosh \left(t^{\prime} / 2\right) & =2 \cosh (a / 2) \cosh (t / 2), \\
t^{\prime} & \leq \ell(\gamma)+2 t,
\end{aligned}
$$

where $a, t$ and $t^{\prime}$ denote the lengths of $\alpha, \tau$ and $\tau^{\prime}$ respectively.
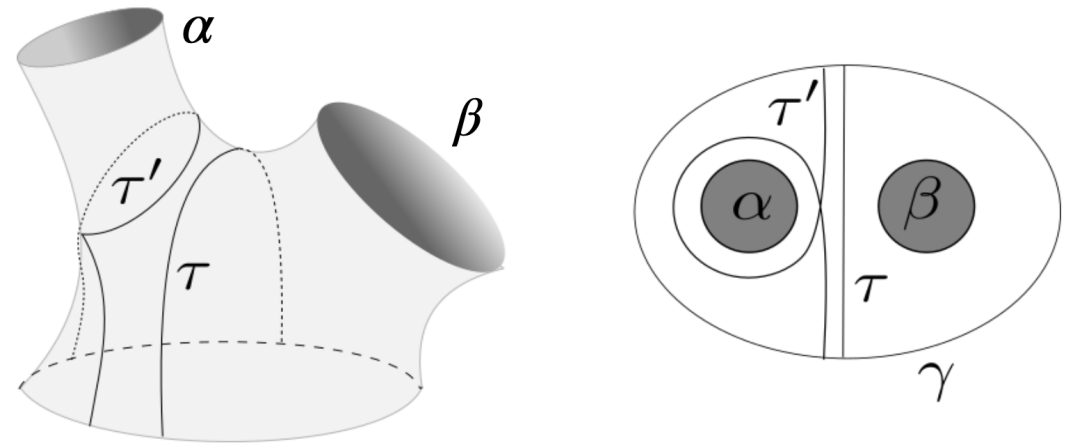

Figure 2

Proof. We cut $P$ along a set of curves to obtain a collection of four right angled pentagons. So cut $P$ along $\tau$, and the shortest ortho geodesics between $\alpha$ and $\gamma$, between $\beta$ and $\gamma$, and $\alpha$ and $\beta$. One of these pentagons has a pair of adjacent edges of lengths $a / 2$ and $b$, the length of the side not adjacent to this pair is $t / 2$ (see Figure 3). By Eq. (1), we have

$$
\cosh (t / 2)=\sinh (a / 2) \sinh (b) .
$$

There is a double cover of the pair of pants such that $\tau^{\prime}$ lifts to a simple curve ${\tilde{\tau^{\prime}}}^{\prime}$ and in this surface there is an embedded right angled pentagon 

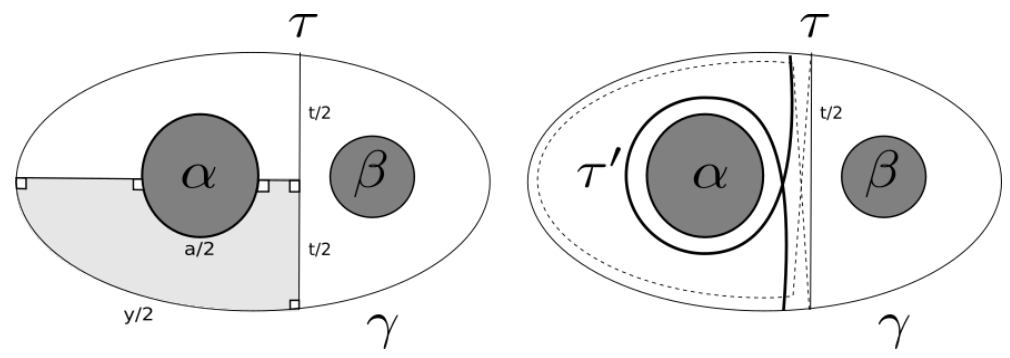

Figure 3

with a pair of adjacent edges of lengths $a$ and $b$ (see Figure 4). The length of the non adjacent edge is $t^{\prime} / 2$.

$$
\cosh \left(t^{\prime} / 2\right)=\sinh (a) \sinh (b) .
$$

By Eq. (5) and Eq. (6), we have

$$
\begin{aligned}
\cosh \left(t^{\prime} / 2\right) & =\sinh (a) \frac{\cosh (t / 2)}{\sinh (a / 2)} \\
& =2 \cosh (a / 2) \cosh (t / 2) .
\end{aligned}
$$

Eq. (4) follows immediately from Figure 3 .
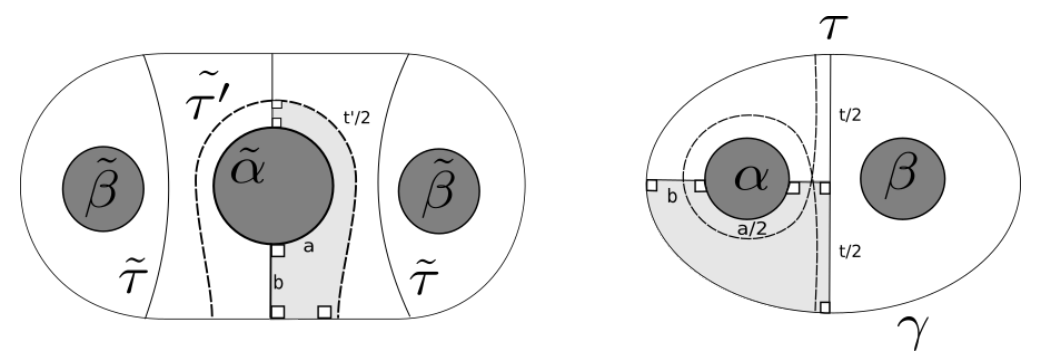

FiguRE 4

\section{Strong RIGIDity FOR THE ONE HOLED TORUS}

We now prove that the ortho spectrum of a one holed torus determines the hyperbolic structure up to isometry. The analogous result for the length spectrum is due to Buser and Semmler [BS88]. For more general surfaces such a strong rigidity result is not possible as one can construct surfaces using abelian covers which are homeomorphic (see Section 6), have the same ortho spectrum but are not isometric. Thus, in the general case we will prove McKean-type theorem for the ortho 
spectrum: that is we show that given a hyperbolic structure on the surface then there are only finitely many hyperbolic metrics with the same ortho spectrum.

Lemma 4.1. Let $\Sigma_{1,1}$ be a hyperbolic one-holed torus with totally geodesic boundary. Let $\alpha$ be the unique simple closed geodesic disjoint from one of the shortest ortho geodesics $\tau$. Then the shortest ortho geodesic that crosses $\alpha$ must meet $\alpha$ in a single point.

Proof. Let $b$ be an ortho geodesic that crosses $\alpha$ more than once. Note that $\ell(\tau) \leq \ell(b)$. We will show that there is an ortho geodesic $x$ which crosses $\alpha$ exactly once and is shorter than $b$.

Let $\widetilde{\Sigma}_{1,1} \rightarrow \Sigma_{1,1}$ denote the infinite cyclic cover in Figure 5 . The
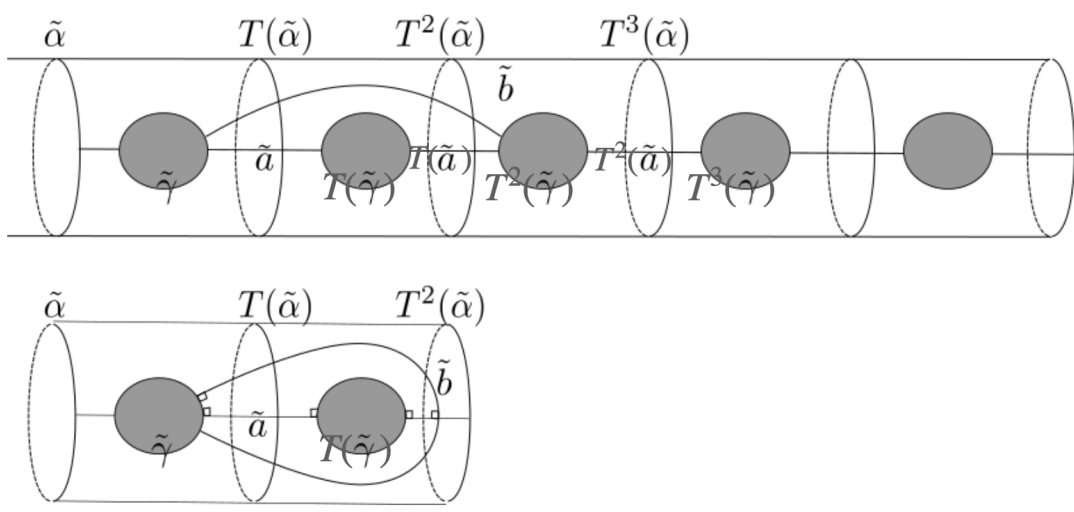

\section{FiguRE 5}

boundary geodesic lifts to a closed geodesic $\tilde{\gamma}$, and $\alpha$ lifts to a closed geodesic $\tilde{\alpha}$. If $T: \widetilde{\Sigma}_{1,1} \rightarrow \widetilde{\Sigma}_{1,1}$ is the generator of the group of deck transformations then $\tilde{\alpha}$ and $T(\tilde{\alpha})$ bound a fundamental domain $D$ for the action of the deck transformations. There is a lift $\tilde{b}$ of $b$ to $\widetilde{\Sigma}_{1,1}$ which, without loss of generality exits the fundamental domain $D$ by crossing $T(\tilde{\alpha})$.

By hypothesis $b$ meets $\alpha$ more than once, so $\tilde{b}$ must meet a translate of $\tilde{\alpha}$ a second time and this translate must be either $T(\tilde{\alpha})$ or $T^{2}(\tilde{\alpha})$. In either case there is an $\operatorname{arc} c$ which minimises the distance between $\tilde{b}$ and $T(\tilde{\gamma})$.

We construct a right angled pentagon with a pair of adjacent edges of length less than $\ell(c), \ell(b) / 2$ and the edge disjoint from this pair is an ortho geodesic $x$ whose projection to $\Sigma_{1,1}$ meets $\alpha$ exactly once. It is always possible to construct the pentagon such that $\ell(c) \leq \ell(\tau) / 2$. 
Now, since cosh and sinh are monotone increasing functions on $\mathbb{R}_{\geq 0}$, by Eq. (1),

$$
\begin{aligned}
\cosh (\ell(x)) & \leq \sinh (\ell(\tau) / 2) \sinh (\ell(b) / 2) \\
& \leq \sinh (\ell(b) / 2)^{2} \\
& <\sinh (\ell(b) / 2)^{2}+\cosh (\ell(b) / 2)^{2} \\
& =\cosh (\ell(b)) .
\end{aligned}
$$

Hence we have $\ell(x)<\ell(b)$.

Theorem 4.2. Let $T_{1}, T_{2}$ be a pair of hyperbolic structures of area $2 \pi$ on the one-holed torus such that the boundary is a closed geodesic. Then $T_{1}$ and $T_{2}$ are isometric if and only if $\mathcal{O}\left(T_{1}\right)=\mathcal{O}\left(T_{2}\right)$.

We begin by using Basmajian's identity to show that boundary lengths are equal then, as in Buser-Semmler, we show that the structures $T_{i}$ determine (essentially) the same Fenchel-Nielsen parameters for a pair of simple geodesics $\alpha_{i}$. More precisely, $\ell\left(\alpha_{1}\right)=\ell\left(\alpha_{2}\right)$ and the twist parameters are the same up to change of sign.

Proof. Let $\gamma$ denote a simple loop freely homotopic to the boundary of the one-holed torus. The free homotopy class of $\gamma$ is invariant under the action of the orientation preserving homeomorphisms of the one-holed torus. By Basmajian Bas93 $\ell(\gamma)$, the length of the unique geodesic in this homotopy class, can be determined from just the ortho spectrum and so it is independent of the choice of structure $T_{i}, i=1,2$.

Let $\tau$ be a simple ortho geodesic and denote its length by $t$. For $i=1,2$ there is a unique simple closed geodesic $\alpha_{i}$ on $T_{i}$ disjoint from $\tau$ and we have

$$
\cosh \left(\ell\left(\alpha_{i}\right) / 2\right)=\sinh (t / 2) \sinh (\ell(\gamma) / 2),
$$

To prove (7) we cut along $\alpha_{i}$ to obtain a pair of pants $P_{i}$ with two boundary geodesics of length $\ell\left(\alpha_{i}\right)$ and another of length $\ell(\gamma)$. There is an embedded right angled pentagon with an adjacent pair of sides of lengths $t / 2, \ell(\gamma) / 2$ and the non adjacent edge has length $\ell\left(\alpha_{i}\right) / 2$ and Eq.(7) follows from Eq.(1).

It follows that if $\ell\left(\tau_{1}\right)=\ell\left(\tau_{2}\right)$ ortho geodesic on $T_{i}$ then the closed simple geodesics $\alpha_{i}$ have the same length. It remains to show that the Fenchel-Nielsen twist parameters are the same for $\alpha_{1}$ and $\alpha_{2}$.

Let $P_{i}$ be the pair of pants we get by cutting along $\alpha_{i}$, and $\mathcal{O}\left(P_{i}, \gamma\right)$ the ortho spectrum of $P_{i}$ of ortho geodesics whose feet are on $\gamma$. Since the geometry of pairs of pants are determined by the boundary lengths, we have $\mathcal{O}\left(P_{1}, \gamma\right)=\mathcal{O}\left(P_{2}, \gamma\right)$. Consider the "set-wise" difference $\mathcal{O}\left(T_{i}\right) \backslash$ 
$\mathcal{O}\left(P_{i}, \gamma\right)$. What we obtain by doing this is the lengths of those ortho geodesics which intersects $\alpha_{i}$ for $i=1,2$. In particular, we can determine $\ell\left(\tau_{i}^{1}\right)$ the length of the shortest such geodesic $\tau_{i}^{1}$ for $i=1,2$. By Lemma 4.1, the ortho geodesic $\tau_{i}^{1}$ meets $\alpha_{i}$ just once. Now any pair of curves that each meets $\alpha_{i}$ just once are related by a Dehn twist, and since the length of an ortho geodesic is strictly convex along a FenchelNielsen twist Ker83 there are at most two possibilities for the choice of $\tau_{i}^{1}$. We normalise the Fenchel-Nielsen twist so that the parameter is 0 when the length of $\tau_{i}^{1}$ is minimal. Using Kerckhoff's formula Ker83] for the variation of length along a Fenchel-Nielsen twist this occurs exactly when $\alpha_{i}$ and $\tau_{i}^{1}$ meet at right angles. It is not difficult to see that when there are two choices for $\tau_{i}^{1}$ this yields a pair of surfaces which are isometric and the corresponding twist parameters differ only in their sign.

\section{Rigidity in General: MCKean's TheOrem}

Sunada Sun85] gave an ingenious geometric construction allowing one to construct pairs of hyperbolic surfaces $Y_{1}, Y_{2} \in \mathcal{M}(\Sigma)$ with the same length spectrum but which are not isometric. On the other hand, by a theorem of McKean McK72], for any given surface $Y \in \mathcal{M}(\Sigma)$ there can only be finitely many surfaces with the same spectrum as $Y$. In this section we prove a version of McKean's theorem for the ortho spectrum:

Theorem 5.1. Let $\Sigma$ be a compact surface with single boundary component $\alpha_{0}$ and $X$ a hyperbolic structure on $\Sigma$ with totally geodesic boundary. Then there are finitely many choices for a hyperbolic structure $Y$ on $\Sigma$ such that $\mathcal{O}(X)=\mathcal{O}(Y)$.

Whilst, as we shall see in Section 6, it is much easier to find pairs of hyperbolic surfaces $Y_{1}, Y_{2} \in \mathcal{M}(\Sigma)$ with the same ortho spectrum but which are not isometric, it turns out that the analogue of McKean's Theorem for the ortho spectrum is quite subtle. So, before giving a proof of Theorem 5.1 in full generality, we discuss the general strategy of the proof and how the Bridgeman-Kahn identity and Poincaré series can be used to obtain the result in two special cases.

5.1. Wolpert's strategy. We will employ a strategy due to Wolpert [Wol77]. His argument is for the length spectrums, however we may almost identically obtain the finiteness as follows. Since the ortho spectrum is discrete, for any compact set $\hat{B}$ of $\mathcal{T}(\Sigma)$ the Teichmüller

space of the surface, the set of hyperbolic structures in $\hat{B}$ with the same 
ortho spectrum as $Y$ is finite. Now ortho spectrum depends only on the point determined by the hyperbolic surface in $\mathcal{M}(\Sigma)$. Thanks to Theorem 2.2 , provided we have given the ortho spectrum, we only need to consider hyperbolic structures on $\Sigma$ with the fixed perimeter. So if $B$ is the projection of $\hat{B}$ to $\mathcal{M}(\Sigma)$ we may apply Mumford's Criterion for pre compactness namely: a subset $B \subset \mathcal{M}(\Sigma)$ is pre compact, if and only if, the infimum of the systole over $B$ is strictly positive. So if we can bound the systole length away from zero, then Mumford's Compactness Theorem implies Theorem 5.1.

5.2. Separating geodesics. Recall that a simple closed curve on a surface is called essential if it is not homotopic to a point, a puncture nor a boundary component. An essential simple closed curve is called separating if it separates the (connected) surface into two components.

We argue by contradiction, suppose that there is a sequence of hyperbolic structures $Y_{i}$ on $\Sigma$ with all boundary components totally geodesic so that $\mathcal{O}\left(Y_{i}\right)=\mathcal{O}(X)$, and that the systole length sys $\left(Y_{i}\right)$ converges to zero. After passing to a subsequence if necessary, we may suppose there is a simple closed curve $\alpha \subset \Sigma$ with $\ell_{Y_{i}}(\alpha) \rightarrow 0$. Suppose that this curve is separating and that one of the subsurfaces has no geodesic boundary component. By Basmajian's identity the perimeter $P$ of $Y_{i}$ does not depend on $i$ so there is some component of the boundary, the good boundary component, for which the length is bounded below away from 0 . We choose $x_{i}$ a base point for $Y_{i}$ on the good boundary component and consider the limit of $\left(Y_{i}, x_{i}\right)$. After passing to a subsequence if necessary we may suppose that this sequence converges to a surface $\left(Y_{\infty}, x_{\infty}\right)$ with at least one cusp and which has, since $\alpha$ is separating, area at most that $Y_{0}$ minus the area of a pair of pants i.e. $2 \pi$. Intuitively, there is a subsurface which is getting further and further from the base point. More concretely, by the Collar Lemma there exists $m$ such that for all $i>m$ there is a collar round $\alpha$ on $Y_{i}$ of width $L$ and as a consequence the length of any ortho geodesic that meets $\alpha$ satisfies

$$
\ell_{Y_{i}}(\tau)>2 L \text {. }
$$

Now by Theorem 2.3 (and Remark 2.4) for each $Y_{i}$

$$
\operatorname{Vol}\left(Y_{i}\right)=\operatorname{Vol}\left(Y_{0}\right)=\sum_{\ell \in \mathcal{O}\left(Y_{0}\right)} F_{2}(\ell)
$$

and so

(1) since each term $F_{2}(\ell)$ is positive and the series is convergent, for any $L>0$ one has

$$
\sharp\left\{\ell<L, \ell \in \mathcal{O}\left(Y_{i}\right)\right\}=\sharp\left\{\ell<L, \ell \in \mathcal{O}\left(Y_{0}\right)\right\}<\infty
$$



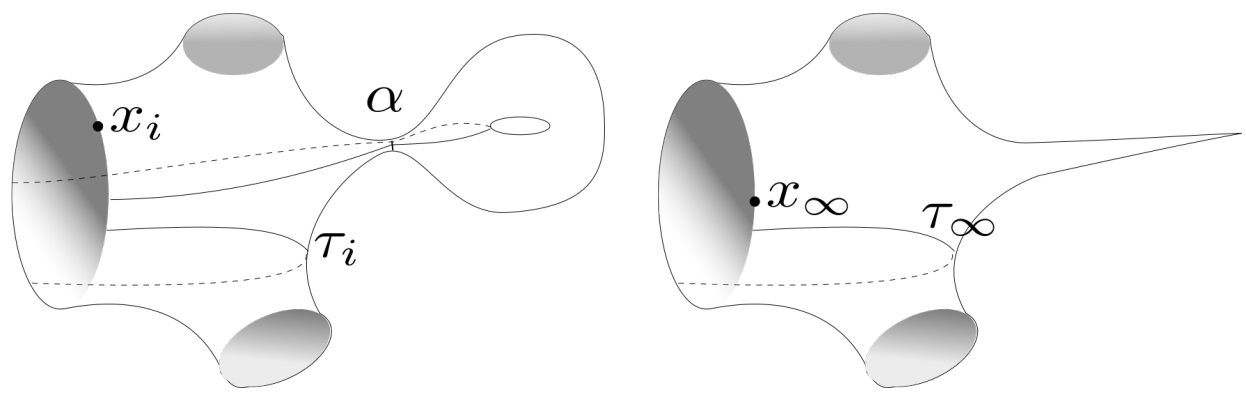

Figure 6. Taking a geometric limit. The curve $\alpha$ has been pinched to a cusp in the limit surface $Y_{\infty}$ on the right. The length of an ortho geodesic that crosses $\alpha$ goes to $\infty$. The sequence of ortho geodesics $\tau_{i}$ limits to $\tau_{\infty}$

(2) there is $L>0$ such that

$$
\sum_{\ell<L} F_{2}(\ell)>\operatorname{Vol}\left(Y_{0}\right)-2 \pi \text {. }
$$

Let $\gamma \subset Y_{i}$ denote the boundary component of $Y_{i}$ containing $x_{i}$. Evidently since the perimeter $P$ of $Y_{i}$ does not depend on $i$ we have a uniform bound on the length of $\gamma$ namely

$$
\ell_{Y_{i}}(\gamma) \leq P
$$

The subset of the component $Y_{i} \backslash\{\alpha\}$ determined by $x_{i}$ containing the ortho geodesics $\tau$ of length less than $L$ is contained in a subset of $Y_{i}$ for which the diameter is bounded by $L+P$. So for $\epsilon>0$ we have uniform bounds, independent of $i$, for the number of balls of radius $\epsilon$ needed to cover the subset of this component which contains ortho geodesics $\tau$ of length less than $L$. Given such a uniform bound it is not difficult to show that for each $\ell \in \mathcal{O}\left(Y_{0}\right) \cap[0, L]$ there is a sequence of ortho geodesics $\tau_{i}(\ell) \subset Y_{i}$ of length $\ell$ which converge to an ortho geodesic $\tau_{\infty}(\ell) \subset Y_{\infty}$ and that reciprocally every such ortho geodesic on $Y_{\infty}$ is obtained in this way. Since, as we have observed above, there are only finitely many ortho geodesics of length less than $L$ on $Y_{i}$ we can pass to a subsequence of $Y_{i}$ for which this convergence is simultaneous that is, for each $\ell \in \mathcal{O}\left(Y_{0}\right) \cap[0, L]$

$$
\tau_{i}(\ell) \rightarrow \tau_{\infty}(\ell) \subset Y_{\infty}
$$

Now we have a contradiction as

$$
\operatorname{Vol}\left(Y_{\infty}\right) \geq \sum_{\ell<L} F_{2}(\ell)>\operatorname{Vol}\left(Y_{0}\right)-2 \pi \geq \operatorname{Vol}\left(Y_{\infty}\right)
$$


In particular, for punctured discs, where every essential simple closed curve is separating and one cut piece never contains boundary, the argument in this Paragraph proves Theorem 5.1.

5.3. Surfaces with small critical exponent. We next observe that under the assumption that the Hausdorff dimension is less than $1 / 2$, we can prove Theorem 5.1 using properties of Poincaré series. From standard calculus it is easy to see that for $x$ large

$$
\sinh ^{-1}\left(\frac{1}{\sinh (x)}\right)=2 e^{-x}+O\left(e^{-3 x}\right),
$$

and, in particular, the following Poincaré series converges for $h=1$ :

$$
\sum_{\alpha^{*}} \exp \left(-h \ell\left(\alpha^{*}\right)\right)
$$

More generally, by work of Patterson, Sullivan and Parker the series converges for all $h$ strictly greater than the critical exponent (dimension of the limit set), see the Appendix for an exposition of this fact.

Theorem 5.2. Let $\delta>0$ and $Y_{n} \in \mathcal{M}(\Sigma)$ be a sequence of compact hyperbolic surfaces with totally geodesic boundary such that the Hausdorff dimension of the limit set (critical exponent) is less than $\frac{1}{2}-\delta$. Then the set of surfaces $Y_{n}$ with the same ortho spectrum as $Y$ is finite.

Proof. We show that the systole is bounded over the set of surfaces $X$ with the same ortho spectrum as $Y$ using the function

$$
\mathcal{M}(\Sigma) \rightarrow \mathbb{R}^{+}, X \mapsto \sum_{\alpha^{*} \subset X} \exp \left(-h \ell\left(\alpha^{*}\right)\right) .
$$

We control the length of the systole using the following observation:

CLAIM: if the systolic length tends to 0 as $n \rightarrow \infty$ then there is a sequence of closed geodesics $\alpha_{n}$ and a sequence of distinct ortho geodesics $\tau_{k}$ and $M>0$ such that:

(1) $\ell\left(\alpha_{n}\right) \rightarrow 0$.

(2) $\ell\left(\tau_{k}\right) \leq k \ell\left(\alpha_{n}\right)-2 \log \left(\ell\left(\alpha_{n}\right)\right)+M$.

Assuming the claim we choose $h<1 / 2$ and strictly greater than the critical exponent. We have

$$
\sum_{\alpha^{*} \subset X} \exp \left(-h \ell\left(\alpha^{*}\right)\right) \geq \sum_{k \geq 0} \exp \left(-h \ell\left(\tau_{k}\right)\right) \geq \frac{C \ell\left(\alpha_{n}\right)^{2 h}}{1-\exp \left(-\ell\left(\alpha_{n}\right)\right)} \sim C \ell\left(\alpha_{n}\right)^{2 h-1}
$$

And thus $\ell\left(\alpha_{n}\right) \not \rightarrow 0$ since $2 h-1<0$ and the Poincaré series converges.

To prove the claim we proceed as follows. We begin by remarking that there is at least one boundary component $\gamma$ say for which $\ell(\gamma) \not \rightarrow 0$. 
For, if all the boundary lengths went to zero then, by the Collar Lemma, the length of the shortest ortho geodesic would tend to infinity and by hypothesis this length is independent of $Y_{n}$. In fact, by Basmajian's identity, we may suppose that

$$
\ell(\gamma) \geq \ell(\partial \Sigma) / N
$$

where $N$ is the number of boundary components.

Let $\alpha_{n}$ be a curve such that $\ell\left(\alpha_{n}\right) \rightarrow 0$. Choose a point on $x \in \gamma$ and construct a piecewise geodesic curve by traveling along the shortest geodesic arc $a_{n}$ to $\alpha_{n}$, going round $\alpha_{n} k$ times, and then returning to $x$ by going back along the shortest route again.

The lengths of these geodesics satisfy

$$
\ell\left(\tau_{k}\right) \leq k \times \ell\left(\alpha_{n}\right)+2 \ell\left(a_{n}\right)
$$

for all $k \geq 0$.

We now show that the length $\ell\left(a_{n}\right)$ is bounded from above by $M / 2-$ $\log \left(\ell\left(\alpha_{n}\right)\right)$ where $M$ depends only on the topology of $\Sigma$. This follows from the fact that for any given $\epsilon>0$ there is a universal constant $M_{\epsilon}$ such that for any $X \in \mathcal{M}(\Sigma)$, the diameter of the $\epsilon$-thick part of $X$ is bounded from above by $M_{\epsilon}$. One can see this by looking at the $\epsilon / 4$-neighbourhood of a diameter realizing path. The neighbourhood must be embedded and the standard hyperbolic geometry shows that the area is diameter $\times 2 \sinh (\epsilon / 4)$. The hyperbolic area of $X$ is $2 \pi|\chi(S)|$ and hence the diameter must be bounded. We may suppose that there exists $\epsilon>0$ such that for any $n, a_{n}$ travels in the $\epsilon$-thick part of $Y_{n}$ until it reaches the collor of $\alpha_{n}$. For otherwise, there must be a sequence of simple closed curves $\alpha_{n}^{\prime} \subset Y_{n}$ with length $\ell\left(\alpha_{n}^{\prime}\right) \rightarrow 0$ as $n \rightarrow \infty$ so that $\alpha_{n}^{\prime}$ intersects with $a_{n}$. In this case we just replace $\alpha_{n}$ with $\alpha_{n}^{\prime}$. Thus we get the constant $M:=2 M_{\epsilon}$ so that the length of $a_{n}$ contained in the $\epsilon$-thick part of $Y_{n}$ is bounded above by $M / 2$. The term $-\log \left(\ell\left(\alpha_{n}\right)\right)$ corresponds to the depth of the collar of $\alpha_{n}$.

5.4. McKean's Theorem in general. We now give a proof of Theorem 5.1 i.e. given a hyperbolic structure on the surface then there are only finitely many hyperbolic metrics with the same ortho spectrum. As before, following Wolpert [Wol77], it suffices to show that the set of surfaces with the same ortho spectrum remains in a pre compact subset of moduli space $\mathcal{M}(\Sigma)$. So, again by Mumford's criterion, we will show that there is a strictly positive lower bound for the systole. Our bound depends on a function which we will call the hyperbolic granulosity of 
a discrete subset $S$ of the positive reals

$$
\operatorname{granulosity}_{S}(L):=\inf \left\{\frac{\cosh (y / 2)}{\cosh (x / 2)} \mid x, y \in S, x<y<L\right\} .
$$

This is a monotone decreasing function of $L \in \mathbb{R}_{+}$, bounded below by 1 and is a measure of the smallest gap in $S$ in the interval $[0, L]$. The hyperbolic granulosity appears naturally from the trigonometric identities we use to determine lengths of closed geodesics from those of ortho geodesics.

Note that the ortho spectrum does not determine the systole's length. For example, in Section 6 we construct pairs of surfaces with the same ortho spectrum but different systolic length. Given such examples, it seems difficult to render such a result effective that is to give an upper bound for the number of such hyperbolic structures which depends only on the topological type of the surface.

Proof of Theorem 5.1. Following Wolpert Wol77]: we suppose that there is an infinite family of pairwise non isometric hyperbolic structures $Y_{n}$ with the same ortho spectrum as $X$. We will show that there is a strictly positive lower bound for the systole.

Let $B$ denote the Bers constant for $\Sigma$. For each $Y_{n}$ choose a pants decomposition $\mathcal{P}_{n}$ which satisfies the $\ell(\gamma) \leq B$ for all $\gamma \in \mathcal{P}_{n}$. We make this choice since this guarantees that if $\ell(\alpha) \rightarrow 0$ as $n \rightarrow \infty$ then $\alpha \in \mathcal{P}_{n}$. We will bound the length $\ell(\gamma)$ from below independently of $n$. This means that $Y_{n}$ remains in a compact subset of moduli space and so must be eventually constant since the ortho spectrum is discrete.

For each $n$ we construct a rooted adjacency graph $\Gamma_{n}$ as follows. The vertices of $\Gamma_{n}$ are the pants in $\mathcal{P}_{n}$, a pair of vertices is joined by an edge if they satisfy the obvious relation and the root of $\Gamma_{n}$ is the vertex corresponding to the pants which meets the boundary. Fix $n$ and choose a rooted spanning tree $T$ for $\Gamma_{n}$. The depth of the spanning tree is bounded above by the number of pants which does not depend on $n$. Let $P$ be a vertex of $T$ we show how to bound the lengths of the boundary geodesics of $P$ by induction on the depth in the spanning tree. The root vertex of $\Gamma_{n}$ is the unique vertex of minimal depth and we begin by this case.

Suppose that $P$ is the root vertex with boundary geodesics $\alpha_{0}, \alpha_{1}$ and $\beta_{1}$, where $\alpha_{0}$ corresponds to the boundary of the surface $\Sigma$. Let $\tau_{0}$ and $\tau_{0}^{\prime}$ are as in Lemma 3.3 with endpoints on $\alpha_{0}$, where we suppose $\tau_{0}^{\prime}$ going around $\alpha_{1}$. Then we have an upper bound for $\ell\left(\tau_{0}\right)$ namely, 
by combining Lemma 3.2 and $x<\sinh x$ for $x>0$

$$
\ell\left(\tau_{0}\right) \leq 2 \operatorname{arcsinh}\left(\frac{2 \exp \left(\ell\left(\alpha_{1}\right) / 2\right)}{\ell\left(\alpha_{0}\right)}\right) \leq 2 \operatorname{arcsinh}\left(\frac{2 \exp (B / 2)}{\ell\left(\alpha_{0}\right)}\right) .
$$

To simplify notation we define a function

$$
F(x):=2 \operatorname{arcsinh}(2 \exp (B / 2) / x)+2 \ell\left(\alpha_{0}\right)
$$

so that

$$
\begin{aligned}
& \ell\left(\tau_{0}\right) \leq F\left(\ell\left(\alpha_{0}\right)\right)-2 \ell\left(\alpha_{0}\right) \\
& \ell\left(\tau_{0}^{\prime}\right) \leq F\left(\ell\left(\alpha_{0}\right)\right)
\end{aligned}
$$

By Lemma 3.3, we have

$$
\cosh \left(\ell\left(\tau_{0}^{\prime}\right) / 2\right)=2 \cosh \left(\ell\left(\alpha_{1}\right) / 2\right) \cosh \left(\ell\left(\tau_{0}\right) / 2\right),
$$

so that $\ell\left(\tau_{0}^{\prime}\right)>\ell\left(\tau_{0}\right)$ and moreover

$$
2 \cosh \left(\ell\left(\alpha_{1}\right) / 2\right)=\frac{\cosh \left(\ell\left(\tau_{0}^{\prime}\right) / 2\right)}{\cosh \left(\ell\left(\tau_{0}\right) / 2\right)}
$$

The ortho spectrum $\mathcal{O}(X)$ is discrete so there are only finitely many distinct values of the ortho spectrum $\mathcal{O}(X)$ less than $F\left(\ell\left(\alpha_{0}\right)\right)$. Thus one can bound $\ell\left(\alpha_{1}\right)$ from below using the hyperbolic granulosity at $F\left(\ell\left(\alpha_{0}\right)\right)$. Note that, by symmetry, this lower bound is also a lower bound for $\ell\left(\beta_{1}\right)$.

Now suppose that we have bounded $\alpha_{n}$ from below and consider the pair of pants with boundary geodesics $\alpha_{n}, \alpha_{n+1}, \beta_{n+1}$. We now construct $\operatorname{arcs} \tau_{n}, \tau_{n}^{\prime}$ (see Figure 7). First join $\alpha_{0}$ and $\alpha_{n}$ by a shortest geodesic $a$, and then connect the endpoint of $a$ on $\alpha_{n}$ and $\alpha_{n+1}$ by a shortest geodesic $a^{\prime}$. The concatenation $a \cup a^{\prime}$ is a path connecting $\alpha_{0}$ and $\alpha_{n+1}$. Then consider the pair of pants $P_{n+1}^{\prime}$ whose boundary curves are $\alpha_{0}, \alpha_{n+1}$ and the boundary of a small neighborhood of $\alpha_{0} \cup \alpha_{n+1} \cup$ $\left\{a \cup a^{\prime}\right\}$. Now we define $\tau_{n}$ and $\tau_{n+1}$ as in Lemma 3.3 applied for $P_{n+1}^{\prime}$ so that

$$
2 \cosh \left(\ell\left(\alpha_{n+1}\right) / 2\right)=\frac{\cosh \left(\ell\left(\tau_{n}^{\prime}\right) / 2\right)}{\cosh \left(\ell\left(\tau_{n}\right) / 2\right)} .
$$

Let $\tau, \tau^{\prime}$ be the ortho geodesics in $P_{n}$ as before. Then the arc $\tau_{n}$ (resp. $\left.\tau_{n}^{\prime}\right)$ is the geodesic representative of a piecewise geodesic constructed by following $a$ to $\alpha_{n}$, traveling round $\alpha_{n}$ to the foot of $\tau$ (resp. $\tau^{\prime}$ ), following $\tau$ (resp. $\tau^{\prime}$ ) then going round $\alpha_{n}$ to the foot of $a$, and finally returning to $\alpha_{0}$ via $a$. There are choices of the direction when we go around $\alpha_{n}$, and we choose the direction so that corresponding geodesic representative $\tau_{n}$ (resp. $\tau_{n}^{\prime}$ ) sits on $P_{n+1}^{\prime}$. 


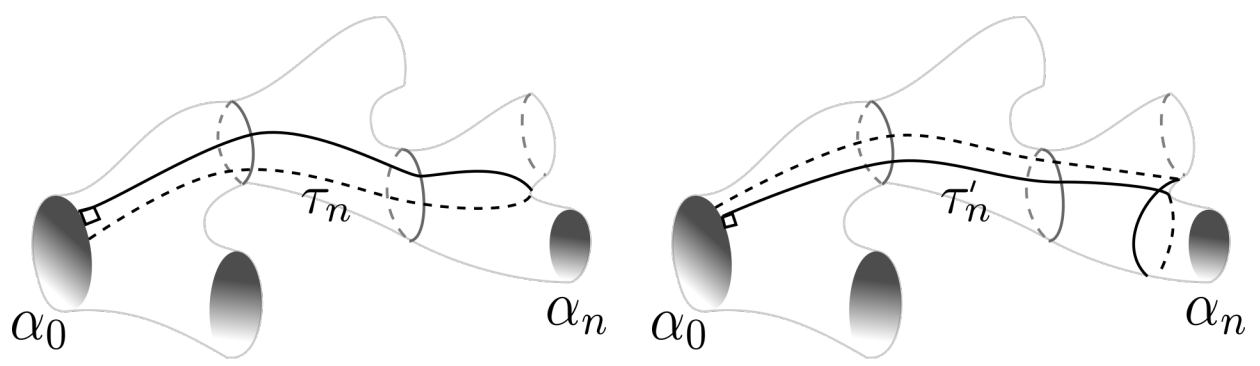

FiguRE 7. The ortho geodesics $\tau_{n}$ and $\tau_{n}^{\prime}$.

Since $\tau_{n}$ is the shortest curve in the homotopy class rel $\partial \Sigma$ its length is bounded above by the piecewise geodesic and we obtain

$$
\ell\left(\tau_{n}\right) \leq 2 \ell(a)+\ell(\tau)+\ell\left(\alpha_{n}\right) \leq 2 \ell(a)+F\left(\ell\left(\alpha_{n}\right)\right) .
$$

Further, by constructing a piecewise geodesic in the same homotopy class as $\tau_{n}^{\prime}$, one sees

$$
\ell\left(\tau_{n}^{\prime}\right) \leq 2 \ell(a)+F\left(\ell\left(\alpha_{n}\right)\right)+B .
$$

Once we bound $\ell(a)$ from above, the same argument as before shows there is a strictly positive lower bound for $\ell\left(\alpha_{n+1}\right)$ which does not depend on $k$.

We now show how to get an upper bound of $\ell(a)$. Given a pair of pants with boundary $\alpha, \beta, \gamma$, we have

$$
\cosh (d(\gamma, \alpha))=\sinh (\ell(\gamma)) \sinh (\ell(\alpha)) \cosh (\ell(\beta))-\cosh (\ell(\gamma)) \cosh (\ell(\alpha))
$$

Hence one obtains an upper bound from $d(\gamma, \alpha)$ from the bounds for the length of $\alpha, \beta$ and $\gamma$. There is a piecewise geodesic curve $\sigma$ homotopic to $a$ obtained by traveling alternately along the shortest ortho geodesic and the arcs of the boundary geodesic of the pants connecting the feet of the ortho geodesics. Then Eq. 15) and upper bound of the length of the pants curve yield an upper bound for $\ell(\sigma)$ and hence for $\ell(a)$.

\section{Abelian COVERS AND THE SPECTRA}

We now give a simple construction which shows that the ortho spectrum fails to distinguish surfaces with different lengths of systole and so it fails to distinguish surfaces which are not isometric. To do this it is convenient to make a special choice for the hyperbolic structure $X$ on $\Sigma_{1,1}$ which will make it easy to determine the length of the systole on degree $n$ covers of $X$. 
For completeness we include the following lemma from which the reader should be able to see why our construction is much simpler than Sunada's.

Lemma 6.1. Let $\tilde{X}$ be an orientable hyperbolic surface with boundary:

(1) If $G$ is group acting freely on $\tilde{X}$. Then the subgroup of $G$ that leaves an ortho geodesic $\tilde{\tau}$ invariant is trivial.

(2) Let $\pi: \tilde{X} \rightarrow X$ be a regular degree $d$ cover of an orientable hyperbolic surface with boundary $X$. If $\tau$ is an ortho geodesic on $X$ then it is covered by exactly $d$ ortho geodesics on $\tilde{X}$.

Proof. The second point follows from the first by taking $G$ to be the group of deck transformations of the cover.

To prove the first point one considers $H$ the stabiliser of $\tilde{\tau}$ in $G$. Any $h \in H$ leaves the end points of $\tilde{\tau}$ invariant and fixes its midpoint, which contradicts the fact that $G$ acts freely.

Lemma 6.2. For any $n>0$ there is a choice of hyperbolic structure $X$ on $\Sigma_{1,1}$ such that:

(1) The systole is shorter than $1 / n$ times the length of the next shortest curve which has length at least $2 \log (1+\sqrt{2})$.

(2) Let $\tilde{X}$ be a degree $n$ cover of $X$ then the systole of the cover has length at most $n \ell(\alpha)$.

Proof. Let $\alpha$ be an essential closed simple curve on $\Sigma_{1,1}$ and $\gamma$ a closed curve homotopic to the boundary $\partial \Sigma_{1,1}$. By the Collar Lemma for any hyperbolic structure on $\Sigma_{1,1}$ such that $\ell(\alpha)$ is sufficiently small the second shortest curve on $\Sigma_{1,1}$ is necessarily a curve $\alpha^{\prime}$ disjoint from $\alpha$. Note that $\alpha^{\prime}$ is not simple since when we cut $\Sigma_{1,1}$ along $\alpha$ we have a pair of pants, and there is no simple closed curves other than the boundary on the pair of pants. It is a result of Hempel [Hem84. Corollary 3.6] that every non-simple has length greater or equal to $2 \log (1+\sqrt{2})$.

Now choose a hyperbolic metric on $P$ such that there is a boundary geodesic of length $\ell(\gamma)$ and the other two boundary geodesics are of length

$$
(1 / n) \times 2 \log (1+\sqrt{2}) .
$$

By identifying these two boundary geodesics one obtains a hyperbolic structure $X$ on $\Sigma_{1,1}$. Then $\ell(\alpha)$ on $X$ equals $2 \log (1+\sqrt{2}) / n$ and the length of $\ell\left(\alpha^{\prime}\right) \geq 2 \log (1+\sqrt{2})=n \ell\left(\alpha^{\prime}\right)$.

Theorem 6.3. Let $k>0$ and set $n=2^{k}$ and $X$ be a hyperbolic structure on $\Sigma_{1,1}$ obtained as in Lemma 6.2. Then there are $k$ cyclic 
covers $\tilde{X}_{i}$ of $X$ such that the length of the systole is different for each of these covers.

Proof. Let $\alpha$ denote the shortest essential closed geodesic on $X$. Since the fundamental group of $\Sigma_{1,1}$ is free on two generators there is a homomorphism onto $\mathbb{Z}$. We choose generators $\alpha, \beta$ for $\pi_{1}\left(\Sigma_{1,1}\right)$ such that $\alpha$ is freely homotopic to $\alpha$ and define a homomorphisms by

$$
\pi_{1}\left(\Sigma_{1,1}\right) \rightarrow \mathbb{Z}, \alpha \mapsto 2^{m}, \beta \mapsto 1
$$

Reducing modulo $2^{k}$ the image of $\alpha$ we obtain a surjective homomorphism

$$
\pi_{1}\left(\Sigma_{1,1}\right) \rightarrow \mathbb{Z} / 2^{k} \mathbb{Z}
$$

and we denote by $\tilde{X}_{m}$ the regular degree $2^{k}$ covering corresponding to the kernel of this homomorphism.

Each closed geodesic $\tilde{\beta}^{\prime} \subset \tilde{X}_{m}$ covers some closed geodesic $\beta^{\prime} \subset X$ and the degree of this cover is the order of the image of $\beta^{\prime}$ in $\mathbb{Z} / 2^{k} \mathbb{Z}$. In particular $\ell\left(\tilde{\beta}^{\prime}\right) \geq \ell\left(\beta^{\prime}\right)$ and if $\beta^{\prime} \neq \alpha$

$$
\ell\left(\tilde{\beta}^{\prime}\right) \geq 2 \log (1+\sqrt{2})>2^{k} \ell(\alpha),
$$

so, since $2^{k}$ is the degree of the cover $\tilde{X}_{m} \rightarrow X$, the shortest closed geodesic on $\tilde{X}_{m}$ must cover $\alpha$.

Finally, we can compute the length of the systole: if $\tilde{\alpha} \subset \tilde{X}_{m}$ covers $\alpha$ then

$$
\ell(\tilde{\alpha})=2^{k-m} \times \ell(\alpha),
$$

so that the systole distinguishes the covers $\tilde{X}_{m}$.

\section{Appendix A. DIMENSION AND ORTHO SPECTRUM IN DIMENSION 3}

Let $M$ be a compact hyperbolic 3-manifold with totally geodesic boundary. We let $\Gamma$ be a Kleinian group such that the convex core of $\mathbb{H}^{3} / \Gamma$ is isometric to $M$. It is observed by several authors [BK10, Cal10] that the ortho spectrum of $M$ determines the volume of $M$ and the area of $\partial M$. In this section, we prove that the Hausdorff dimension of the limit set $\Lambda(\Gamma)$ of $\Gamma$ can also be determined by the ortho spectrum of $M$. Since the boundary of $M$ is totally geodesic and hence the limit sets of the boundary subgroups are round circles, the Ahlfors' finiteness theorem Ahl64 implies that the limit set $\Lambda(\Gamma)$ is a circle packing of $\partial \mathbb{H}^{3}=\mathbb{C} \cup\{\infty\}$. By considering conjugate if necessary, we may assume that $\infty \in \partial \mathbb{H}^{3} \backslash \Lambda(\Gamma)$. First, we recall the work of Parker. Let $\mathcal{C}$ be a circle packing of $\mathbb{C}=\partial \mathbb{H}^{3} \backslash\{\infty\}$. When we discuss the radius of circles in $\mathcal{C}$, we consider the Euclidean metric on $\mathbb{C}$. Let $\mathcal{R}(\mathcal{C})$ denote the set 
of radii of the circles in $\mathcal{C}$ counted with multiplicity. Then the circle packing exponent of $\mathcal{C}$ is

$$
e_{\mathcal{C}}:=\inf \left\{t: \sum_{r \in \mathcal{R}(\mathcal{C})} r^{t}<\infty\right\}
$$

Parker showed the following.

Theorem A.1 ([Par95]). Let $\Gamma$ be as above. Suppose $\infty \in \partial \mathbb{H}^{3} \backslash$ $\Lambda(\Gamma)$. Then the circle packing exponent e of $\Lambda(\Gamma)$ equals the Hausdorff dimension $d$ of $\Lambda(\Gamma)$.

We now define the exponent of ortho spectrum as follows. Let $\mathcal{O}(M)$ denote the ortho spectrum of $M$. Then we define the ortho spectrum exponent by

$$
e_{\mathcal{O}}:=\inf \left\{t: \sum_{l \in \mathcal{O}(M)} \frac{1}{e^{t l}}<\infty\right\} .
$$

Our goal is the following.

Theorem A.2. Let $M$ and $\Gamma$ be as above. Suppose $\infty \in \partial \mathbb{H}^{3} \backslash \Lambda(\Gamma)$. Then the ortho spectrum exponent $e_{\mathcal{O}}$ of $M$ equals the Hausdorff dimension $d$ of $\Lambda(\Gamma)$.

Proof. Let $\gamma$ be a circle in $\Lambda(\Gamma)$ and $H<\Gamma$ be the (setwise) stabiliser of $\gamma$. By applying conjugate if necessary, we may suppose that $\gamma$ is the unit circle in $\mathbb{C}$. As $H$ acts on the unit disc surrounded by $\gamma$ discontinuously, we have a fundamental domain $D$ which contains 0 . First we claim that there is a constant $C>0$ which depends only on $M, H$ and $D$ with the following property. Let $\delta$ be a circle in $\Lambda(\Gamma)$ meeting $D$, we let $l$ denote the length of the ortho geodesic connecting $\delta$ and $\gamma$. Let us show that the radius $r$ of $\delta$ satisfies

$$
\frac{1}{C e^{l}} \leq r \leq \frac{1}{e^{l}}
$$

The ortho geodesic connecting $\delta$ and $\gamma$ lies on a totally geodesic hyperplane intersecting with $\delta$ and $\gamma$. Hence it suffices to discuss the case of dimension two, and we now suppose $\gamma=\{-1,1\} \subset \partial \mathbb{H}^{2}$. The convex hull $C(\gamma) \subset \mathbb{H}^{2}$ of $\gamma$ is the half circle of radius 1 centred at 0 . Let $\delta^{\prime} \subset \mathbb{H}^{2}$ denote the half circle centred at 0 with hyperbolic distance $l$ from $C(\gamma)$. The radius $r^{\prime}$ of $\delta^{\prime}$ equals $1 / e^{l}$. Since the $l$-neighbourhood (in hyperbolic metric) of the geodesic $\gamma$ becomes thickest (in terms of Euclidean metric) at the top, among geodesics of distance $l$ apart from $\gamma, \delta^{\prime}$ has the largest possible radius. Hence we have $r \leq 1 / e^{l}$. We now consider an isometry $A=\left(\begin{array}{cc}s & t \\ u & v\end{array}\right) \in \operatorname{PSL}(2, \mathbb{R})$ which preserves 
$C(\gamma)$ and maps $\delta^{\prime}$ to $\delta$. Without loss of generality, we may suppose $s>0$. Since $A$ preserves -1 and 1 , we have $s=v$ and $t=u$, and hence, $s^{2}-t^{2}=1$. Then the centre of $\delta$ is $t / s$. Note that since $M$ is compact and there is a lower bound of the length of ortho geodesics, there exists $1>\epsilon>0$ which depends only on $M, H$ and $D$ such that $\partial \delta \subset(-1+\epsilon, 1-\epsilon)$ whenever $\delta$ meets $D$. A simple calculation shows that the radius $r$ of $\delta$ equals

$$
\frac{r^{\prime}}{s\left(t r^{\prime}+s\right)} .
$$

Since $t / s \leq 1-\epsilon$ and $s^{2}-t^{2}=1$, we have $t \leq(1-\epsilon) s$, and

$$
s^{2} \leq \frac{1}{1-(1-\epsilon)^{2}} .
$$

Therefore as $r^{\prime} \leq 1-\epsilon$, and $r^{\prime}=1 / e^{l}$, we have

$$
r=\frac{1 / e^{l}}{s\left(t r^{\prime}+s\right)} \geq \frac{1 / e^{l}}{s^{2}\left((1-\epsilon) r^{\prime}+1\right)} \geq \frac{1-(1-\epsilon)^{2}}{1+(1-\epsilon)^{2}} \cdot \frac{1}{e^{l}},
$$

and the claim follows.

Now we prove $e_{\mathcal{O}} \leq d$. By Theorem A.1. we have $e_{\Lambda(\Gamma)}=d$. For a subset $T \subset \partial \mathbb{H}^{3}$, let

$$
e_{T}:=\inf \left\{t: \sum_{r: \text { radius of circles meeting } T} r^{t}<\infty\right\} .
$$

Then we have $e_{T} \leq e_{\Lambda(\Gamma)}=d$ for any $T$, in particular when $T=D$. Let $B \subset \partial M$ be the component corresponding to $H$, and $\mathcal{O}_{B}$ denote the set of lengths of ortho geodesics of $M$ one of whose endpoints lies on $B$. Then each element in $\mathcal{O}_{B}$ corresponds to an ortho geodesic which has a lift in $\mathbb{H}^{3}$ so that it connects $\gamma$ and a circle meeting $D$. As there are only finitely many components in $\partial M$, we may suppose

$$
e_{B}:=\inf \left\{t: \sum_{l \in \mathcal{O}_{B}} \frac{1}{e^{t l}}<\infty\right\}
$$

equals $e_{\mathcal{O}}$. Since the constant multiplicable error in inequality (16) does not change the critical exponents, we have $e_{B}=e_{D}$ and hence $e_{D}=e_{\mathcal{O}}$. Hence we have $e_{\mathcal{O}} \leq d$.

For the converse inequality, we use the work of Larman Lar66 which says that for any compact subset $K \subset \mathbb{C}$ and a circle packing $\mathcal{C}$ of $K$, the circle packing exponent of $\mathcal{C}$ is greater than or equal to the Hausdorff dimension of $\mathcal{C}$. We first take large enough ball $K^{\prime}$ centred at 0 and contained in $\gamma$. Then we let $K$ to be the subspace obtained by removing all circles intersecting with $\partial K^{\prime}$ from $K^{\prime}$. By taking $K^{\prime}$ large enough we may suppose that $K$ contains all the circles meeting 
$D$. As $D$ is a fundamental domain and $K$ is compact, we may cover $K$ by finitely many translates $\gamma_{i} D$ of $D$ where $\gamma_{i} \in \Gamma$. By the same argument as above we have $e_{\gamma_{i} D}=e_{\mathcal{O}}$ for every $i$, which implies

$$
e_{\cup_{i} \gamma_{i} D}=e_{\mathcal{O}} \text {. }
$$

Hence we have $e_{\mathcal{O}}=e_{D}=e_{\cup_{i} \gamma_{i} D}=e_{K}$, and the Hausdorff dimension of the restriction of $\Lambda(\Gamma)$ on $K$ equals $d$. Therefore we have $e_{\mathcal{O}}=e_{K} \geq d$ by Larman. Thus $e_{\mathcal{O}}=d$ follows.

\section{REFERENCES}

[Ahl64] Lars V Ahlfors, Finitely generated Kleinian groups, American Journal of Mathematics 86 (1964), no. 2, 413-429.

[Bas93] Ara Basmajian, The orthogonal spectrum of a hyperbolic manifold, Amer. J. Math. 115 (1993), no. 5, 1139-1159.

[Bea95] Alan F. Beardon, The geometry of discrete groups, Graduate Texts in Mathematics, vol. 91, Springer-Verlag, New York, 1995. Corrected reprint of the 1983 original.

[Bri11] Martin Bridgeman, Orthospectra of geodesic laminations and dilogarithm identities on moduli space, Geom. Topol. 15 (2011), no. 2, 707-733.

[BD07] Martin Bridgeman and David Dumas, Distribution of intersection lengths of a random geodesic with a geodesic lamination, Ergodic Theory Dynam. Systems 27 (2007), no. 4, 1055-1072.

[BK10] Martin Bridgeman and Jeremy Kahn, Hyperbolic volume of manifolds with geodesic boundary and orthospectra, Geom. Funct. Anal. 20 (2010), no. 5, 1210-1230.

[Bus10] Peter Buser, Geometry and spectra of compact Riemann surfaces, Modern Birkhäuser Classics, Birkhäuser Boston, Inc., Boston, MA, 2010. Reprint of the 1992 edition.

[BS88] P. Buser and K.-D. Semmler, The geometry and spectrum of the one-holed torus, Comment. Math. Helv. 63 (1988), no. 2, 259-274.

[Cal10] Danny Calegari, Chimneys, leopard spots and the identities of Basmajian and Bridgeman, Algebr. Geom. Topol. 10 (2010), no. 3, 1857-1863.

[Gor89] Carolyn S. Gordon, When you can't hear the shape of a manifold, Math. Intelligencer 11 (1989), no. 3, 39-47, DOI 10.1007/BF03025190. With an appendix by Dennis DeTurck. MR1007037

[Hem84] John Hempel, Traces, lengths, and simplicity for loops on surfaces, Topology Appl. 18 (1984), no. 2-3, 153-161, DOI 10.1016/0166-8641(84)900075. MR769288

[Ker83] Steven P. Kerckhoff, The Nielsen realization problem, Ann. of Math. (2) 117 (1983), no. 2, 235-265, DOI 10.2307/2007076. MR690845

[MM13] Hidetoshi Masai and Greg McShane, Equidecomposability, volume formulae and orthospectra, Algebr. Geom. Topol. 13 (2013), no. 6, 3135-3152.

[MM] Hidetoshi Masai and Greg McShane, Isospectral configurations in Euclidean and Hyperbolic Geometry, in preparation.

[McK72] H. P. McKean, Selberg's trace formula as applied to a compact Riemann surface, Comm. Pure Appl. Math. 25 (1972), 225-246. 
[Lar66] D. G. Larman, On the exponent of convergence of a packing of spheres, Mathematika 13 (1966), 57-59.

[Par18] Hugo Parlier, Interrogating surface length spectra and quantifying isospectrality, Math. Ann. 370 (2018), no. 3-4, 1759-1787.

[Par95] John R. Parker, Kleinian circle packings, Topology 34 (1995), no. 3, 489496.

[Sun85] Toshikazu Sunada, Riemannian coverings and isospectral manifolds, Ann. of Math. (2) 121 (1985), no. 1, 169-186, DOI 10.2307/1971195. MR782558

[Wol77] Scott Wolpert, The eigenvalue spectrum as moduli for compact Riemann surfaces, Bull. Amer. Math. Soc. 83 (1977), no. 6, 1306-1308.

Department of Mathematics Tokyo Institute of Technology 2-12-1, Ookayama, Meguro-Ku, TOKYO. 152-8551. Japan

Email address: masai at math.titech.ac.jp

Institut Fourier 100 Rue des maths, BP 74, 38402 St Martin D'Hères CEDEX, France

Email address: mcshane at univ-grenoble-alpes.fr 